\title{
Graphene reinforced ultra high molecular weight polyethylene with improved tensile strength and creep resistance properties
}

\author{
A. Bhattacharyya ${ }^{1 *}$, S. Chen ${ }^{2}, M . Z h u^{2}$ \\ ${ }^{1}$ Nanotech Research Facility, PSG Institute of Advanced Studies, 641004 Coimbatore, India \\ ${ }^{2}$ College of Materials Science and Engineering, Donghua University, 201620 Shanghai, China
}

Received 9 August 2013; accepted in revised form 24 September 2013

\begin{abstract}
Reduced graphene oxide or graphene was dispersed in ultra high molecular weight polyethylene (UHMWPE) using two methods to prepare nanocomposite films. In pre-reduction method, graphite oxide (GO) was exfoliated and dispersed in organic solvents and reduced to graphene before polymer was added, while reduction of graphene oxide was carried out after polymer addition for in situ reduction method. Raman spectroscopic study reveals that the second method results in better exfoliation of graphene but it has more amorphous content as evident from selected area electron diffraction (SAED) pattern, wide angle X-ray and differential scanning calorimetry (DSC). The nanocomposite film produced by prereduction method possesses higher crystallinity (almost the same as that of the pure film) as compared to the in situ method. It shows better modulus (increased from 864 to $1236 \mathrm{MPa}$ ), better strength (increased from 12.6 to 22.2 MPa), network hardening and creep resistance (creep strain reduced to $9 \%$ from $50 \%$ when $40 \%$ of maximum load was applied for $72 \mathrm{~h}$ ) than the pure film. These findings show that graphene can be used for reinforcement of UHMWPE to improve its tensile and creep resistance properties.
\end{abstract}

Keywords: nanocomposites, mechanical properties, graphene, ultra high molecular weight polyethylene (UHMWPE)

\section{Introduction}

Ultra high molecular weight polyethylene (UHMWPE) has been extensively used for various high performance application areas as biomedical, defence, etc $[1,2]$. However, its adverse properties such as creep, abrasion resistance, etc. are still a matter of concern [3]. Several research groups have observed that the mechanical properties of a polymer can be improved using small amount of nanofillers in it [4]. Among the nanofillers, carbon nanotube (CNT) is researched most as a reinforcement due to its excellent tensile properties [5]. However, after years of research, the expected improvements have not yet been achieved due to several reasons. Except the difficulty of dispersing CNT in viscous solution, the caged structure of CNT is the main drawback for load transmission from polymer matrix to the reinforcement as it slips from the polymer matrix [6]. Functionalization of CNT partially solves this problem, but it destroys the caged structure which leads to drop in tensile properties [7]. In this aspect, graphene or functionalized graphene has the potential to supersede CNT. The measured and theoretical properties of graphene are comparable with CNT [8], moreover, due to its flat structure, the strength translation towards matrix at different direction is possible (CNT has good tensile property only along its length) and matrix slippage problem can be minimized. Most of the groups working with graphene based polymer nanocom-

\footnotetext{
${ }^{*}$ Corresponding author, e-mail: amitbha1912@gmail.com (C) BME-PT
} 
posites found improvements in mechanical properties $[9,10]$. The mechanical and thermo mechanical properties of graphene oxide reinforced poly lacticco-glycolic acid nanofiber mesh are significantly enhanced when only 1 and $2 \mathrm{wt} \%$ of graphene oxide were dispersed in it [11]. In a review on graphene based nanocomposites, Sengupta et al. [12] listed few graphene and high density polyethylene (HDPE) based nanocomposites showing increase in mechanical properties. Jiang and Drzal [13] found that the paraffin wax modification on graphene results in improved electrical conductivity and flexural properties than uncoated one for HDPE based nanocomposites. However, more research is required in this field to understand the interaction of graphene with polymer matrix. The basic problems are the dispersion and orientation of mono or few layered graphene in the polymer matrix and the interface between polymer matrices with graphene so that efficient load transfer is possible. The strength realization using graphene is not adequate till now; mainly because of the lack of proper inter-phase between graphene and polymer and due to the poor 'between-plane' strength of graphene causes slippage of multilayer graphene [12]. For these, graphene preparation, dispersion in polymer matrix and matrixgraphene load transfer need to be investigated. In this paper, reduced graphene oxide dispersed UHMWPE nanocomposite films are prepared in two different process routes. The produced films are characterized to correlate with their tensile and time dependant creep strain properties.

\section{Experimental}

\subsection{Material}

Graphite having particle size less than $10 \mu \mathrm{m}$ was procured from Shanghai Yifan Graphite Co Ltd., China. UHMWPE powder having average molecular weight of $3 \times 10^{6}$ was supplied by Shanghai Lianle Chemical Co. Ltd., China. Ortho-dichlorobenzene (ODCB) and N, N'-dimethylformamide (DMF) were purchased from Aladdin Industrial Corporation and Shanghai Boer Chemical Reagent Co. Ltd., China, respectively. All other chemicals were supplied by Sinopharm Chemical Reagent Co. Ltd., China and used as received without further purification.

\subsection{Preparation of graphite oxide (GO)}

GO has been synthesized using Hummers method from graphite [14]. In short, $5 \mathrm{~g}$ of graphite and $10 \mathrm{~g}$ of sodium nitrate $\left(\mathrm{NaNO}_{3}\right)$ were added to $150 \mathrm{~mL}$ of concentrated sulfuric acid $\left(\mathrm{H}_{2} \mathrm{SO}_{4}\right)$ with continuous stirring $(500 \mathrm{rpm})$ for $30 \mathrm{~min}$. The mixture was kept in ice bath to keep the temperature low and $15 \mathrm{~g}$ of potassium permanganate $\left(\mathrm{KMnO}_{4}\right)$ was added very slowly in it (extreme care has been taken as it is a highly exothermic reaction). The mixture was subjected to ultrasonic treatment $(42 \mathrm{kHz})$ for $30 \mathrm{~min}$ and then kept for $3 \mathrm{~h}$ with stirring (500 rpm). It was carefully mixed with $200 \mathrm{~mL}$ de-ionized water to stop the reaction. $50 \mathrm{~mL}$ hydrogen peroxide $\left(\mathrm{H}_{2} \mathrm{O}_{2}\right)$ was added to reduce the un-reacted $\mathrm{KMnO}_{4}$. The mixture was centrifuged and washed twice with dilute hydrochloric acid. Then it was washed three times with de-ionized water and filtered with $0.2 \mu \mathrm{m}$ acetate filter. The resulting GO was dried at $40^{\circ} \mathrm{C}$ for $60 \mathrm{~h}$. To test the graphene yield, $100 \mathrm{mg}$ GO was exfoliated and dispersed in de-ionized water with $30 \mathrm{~min}$ ultrasonic treatment and $1 \mathrm{~h}$ stirring (500 rpm) and $0.5 \mathrm{~mL}$ of phenylhydrazine was added at $90^{\circ} \mathrm{C}$ for reduction of graphene oxide. The reaction was allowed to continue for $4 \mathrm{~h}$. After that, graphene was filtered, washed twice and dried for $4 \mathrm{~h}$. The yield was approximately $60 \mathrm{wt} \%$. However, for nanocomposites preparation, graphite oxide (GO) taken on weight basis was reported.

\subsection{Preparation of nanocomposites}

As solvent of UHMWPE and graphene dispersion media, 1:4 (v/v) mixtures of DMF and ODCB were selected. Graphene oxide and graphene forms stable dispersion in DMF [15] but DMF cannot dissolve UHMWPE. ODCB is a good solvent for UHMWPE, as well as CNT can disperse reasonably well in it [16]. Exfoliation of graphite in ODCB has also been reported [17]. So, this solvent was chosen. However, it has been observed that the exfoliation and dispersion of GO is not very good in ODCB. For this, a combination solvent system of DMF and ODCB was used. Two routes were followed for dispersion of reduced graphene oxide in polymer:

Route 1 (R-1) or the pre-reduction method: $1 \mathrm{wt} \%$ (on weight of UHMWPE) GO was exfoliated and dispersed in DMF and ODCB (1:4 ratio) by ultrasonic treatment $(30 \mathrm{~min})$, followed by reduction with phenylhydrazine for $4 \mathrm{~h}$ with continuous stirring (500 rpm). Subsequently, 3\% (w/v on total solvent taken) UHMWPE powder was added slowly at $140^{\circ} \mathrm{C}$ with continuous stirring for $2 \mathrm{~h}$ till it completely dissolved. 
A variation of R-1 was also tried as follows: GO was exfoliated in DMF, followed by reduction with phenylhydrazine for $4 \mathrm{~h}$ with continuous stirring (500 rpm). UHMWPE powder was added slowly at $140^{\circ} \mathrm{C}$ in ODCB (taken separately as 4 times of DMF on volume basis) with continuous stirring (500 rpm) for $2 \mathrm{~h}$ till it was completely dissolved. Subsequently, the graphene dispersion was added into it. However, as viscosity was very high, this process did not give good dispersion of graphene in polymer solution, so it was not used.

Route 2 (R-2) or in situ reduction method: $1 \mathrm{wt} \%$ (on weight of UHMWPE) GO was exfoliated and dispersed in DMF and ODCB (1:4 ratio) with $30 \mathrm{~min}$ ultrasonic treatment and $1 \mathrm{~h}$ stirring $(500 \mathrm{rpm})$, followed by addition of small amount of UHMWPE (twice the weight of GO) at $140^{\circ} \mathrm{C}$, stirring continued till the polymer dissolved, followed by reduction with phenylhydrazine for $4 \mathrm{~h}$. Subsequently, remaining UHMWPE powder was added slowly at $140^{\circ} \mathrm{C}$ with continuous stirring for $2 \mathrm{~h}$ till it completely dissolved. The dispersion contained total 3\% UHMWPE (w/v on total solvent taken).

Schematic representations of both the process routes are described in Figure 1. In the case of route 2 or in situ reduction, Small amount of polymer is added in the system before reduction as reduced graphene oxide tends to re-agglomerate in the media. The added polymer is expected to hinder the graphene agglomeration after reduction. The total amount of UHMWPE has not been added as the viscosity will be very high so dispersion would be difficult and create disturbance in reduction process. Again, the long stirring for $4 \mathrm{~h}$ during graphene oxide reduction would lead to gel break down of UHMWPE [18].

In both the process routes, after complete dissolution, the polymer-graphene dispersions were cast into films and dried at $70^{\circ} \mathrm{C}$ for $48 \mathrm{~h}$. The average thickness of films was found to be $60 \mu \mathrm{m}$.

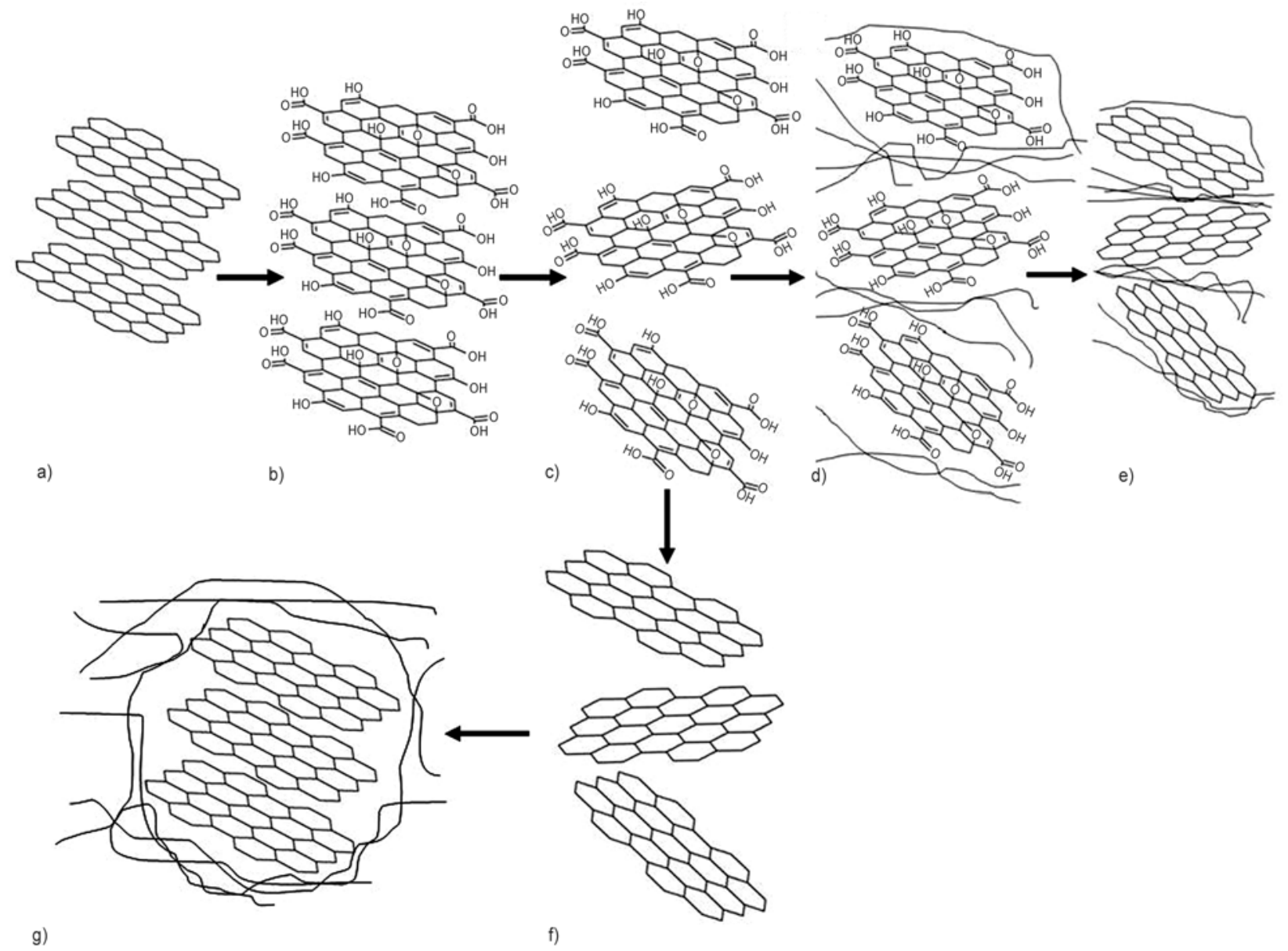

Figure 1. Schematic representation of process routes starting from graphite (a), graphite oxide (b), graphene oxide (c), polymer chains around graphene oxide as in route 2 (d), polymer coated graphene after in situ reduction in route $2(\mathrm{e})$, pre-reduction of graphene in route 1 (f) and graphene embedded in polymer as in route $1(\mathrm{~g})$ 


\subsection{Characterization}

Raman spectroscopic studies were carried out in $514 \mathrm{~nm}$ laser source of inVia Raman spectroscope from Renishaw, UK. High resolution transmission electron microscope (HRTEM model: JEM 2100) from Jeol Inc., Japan was used for imaging and selected area electron diffraction (SAED) studies of graphene and graphene oxide (at $200 \mathrm{kV}$ ) and nanocomposite films (at $100 \mathrm{kV}$ ). X ray powder diffractometer (D/Max-2550 PC made by Rigaku, Japan) was used to register the X-ray patterns of the particles. Differential scanning calorimeter (DSC) tests were carried out in DSC Q20 produced by TA instruments at a scanning rate of $10^{\circ} \mathrm{C} / \mathrm{min}$. Instron 4206 universal tester by Instron Engineering Corp. USA was used for tensile tests. Sample strips with $2 \mathrm{~mm}$ width have been cut and 10 samples were tested in each case at a speed of $5 \mathrm{~mm} / \mathrm{min}$ with a jaw separation distance of $20 \mathrm{~mm}$. The tensile modulus was calculated at initial straining of 0.5 to $0.7 \%$. Maximum strain at break was measured as percentage ratio of extension to initial test length (jaw separation distance). For creep tests, the film strips of same dimension were loaded with 20, 40, 60 and $80 \%$ of their average maximum strength. The strains as percentage ratio of extension to initial test length of $20 \mathrm{~mm}$ have been recorded at different test time. Four samples were tested in each case. Both the tensile and creep tests were carried out at laboratory condition of $27^{\circ} \mathrm{C}$ and $65 \%$ relative humidity.

\section{Results and discussions \\ 3.1. Characterization of graphene-UHMWPE nanocomposites}

\subsubsection{Raman spectroscopy}

Figure 2 illustrates the Raman spectrum of graphite, graphene, UHMWPE film and two nanocomposite films made by two different process routes. D $\left(\sim 1330 \mathrm{~cm}^{-1}\right)$ and $\mathrm{G}\left(\sim 1580 \mathrm{~cm}^{-1}\right)$ band peaks of graphite and graphene are shown. The D peak, exists in defected graphene, is due to first order zone boundary phonons. The chemically converted graphene includes significant amount of defects which causes enhanced relative intensity of $\mathrm{D}$ band with respect to $G$ band [17]. The relative peak intensity of $D$ band to $\mathrm{G}$ band is often used to estimate the amount

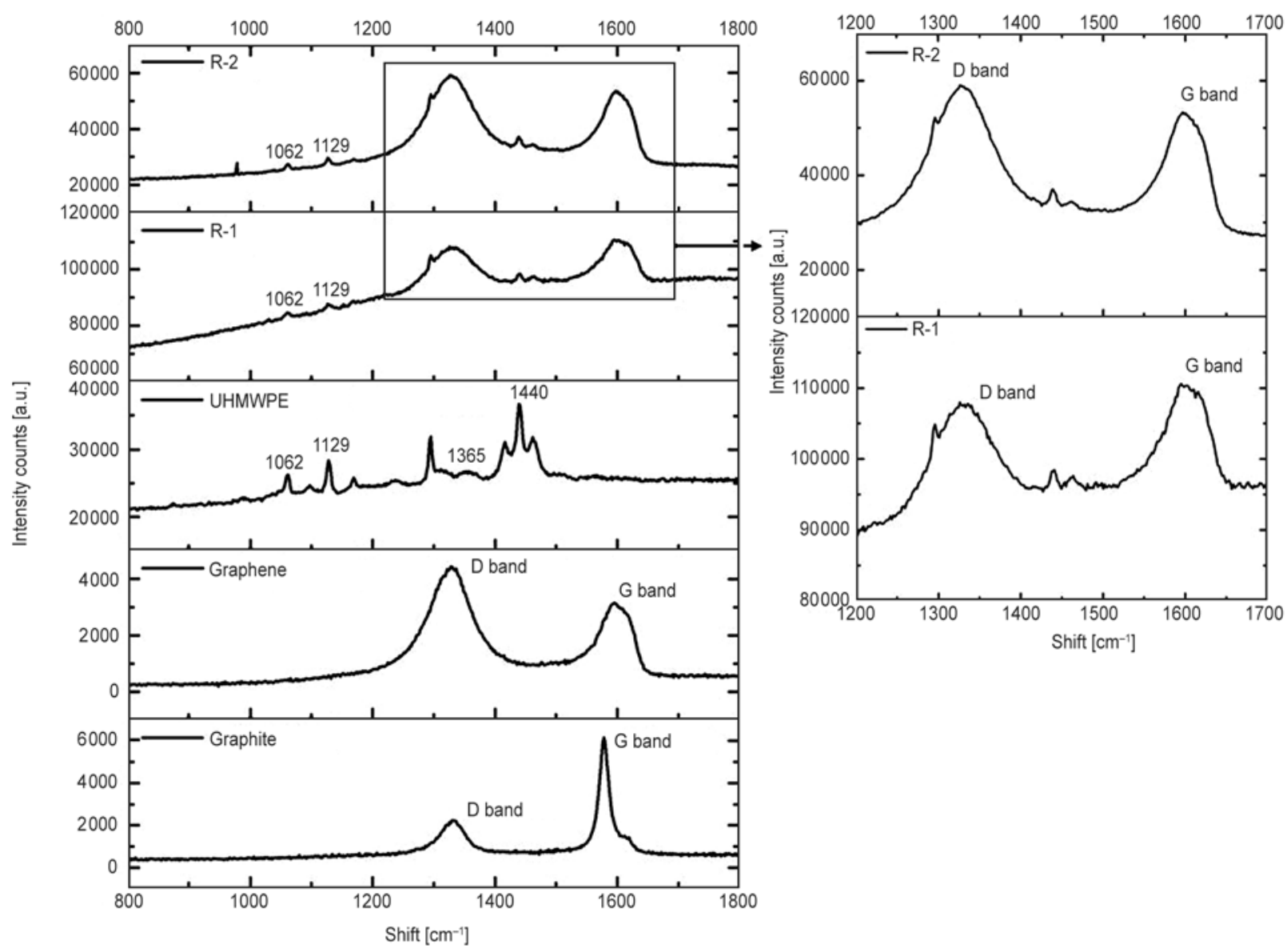

Figure 2. Raman spectra for graphite, synthesized graphene, UHMWPE film and graphene/UHMWPE nanocomposite films in route $1(\mathrm{R}-1)$ and route $2(\mathrm{R}-2)$ 
of defects in carbon materials. The $\mathrm{G}$ band to $\mathrm{D}$ band ratio gives an idea of in-plane crystallite size ( $L a$ ) [19]. For the reduced graphene oxide synthesized in this work, it is approximately $2.7 \mathrm{~nm}$. The $\mathrm{C}-\mathrm{C}$ asymmetric and symmetric stretching bands (1062 and $1129 \mathrm{~cm}^{-1}$ ) of UHMWPE lie at the same position for both the nanocomposite films produced by R-1 and R-2 as shown in Figure 2. This proves the unstressed condition of nanocomposite films or the stress levels are same as the pure film [20]. The relative peak intensities of $\mathrm{G}$ and $\mathrm{D}$ band peaks of graphene differ in the nanocomposite films produced by two routes. After baseline correction, the relative peak intensity of $G$ band to $D$ band of

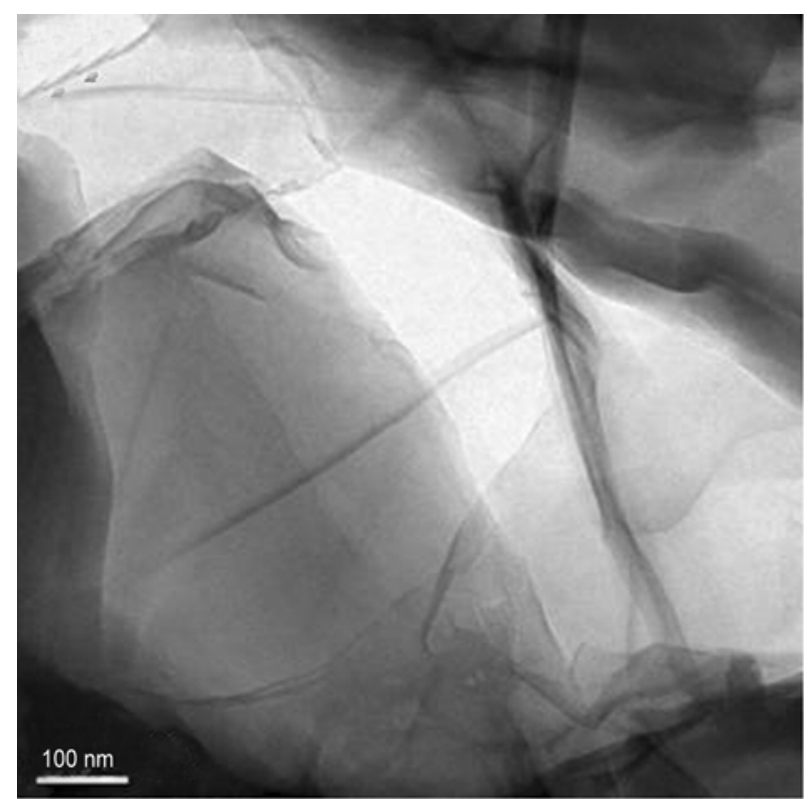

a)

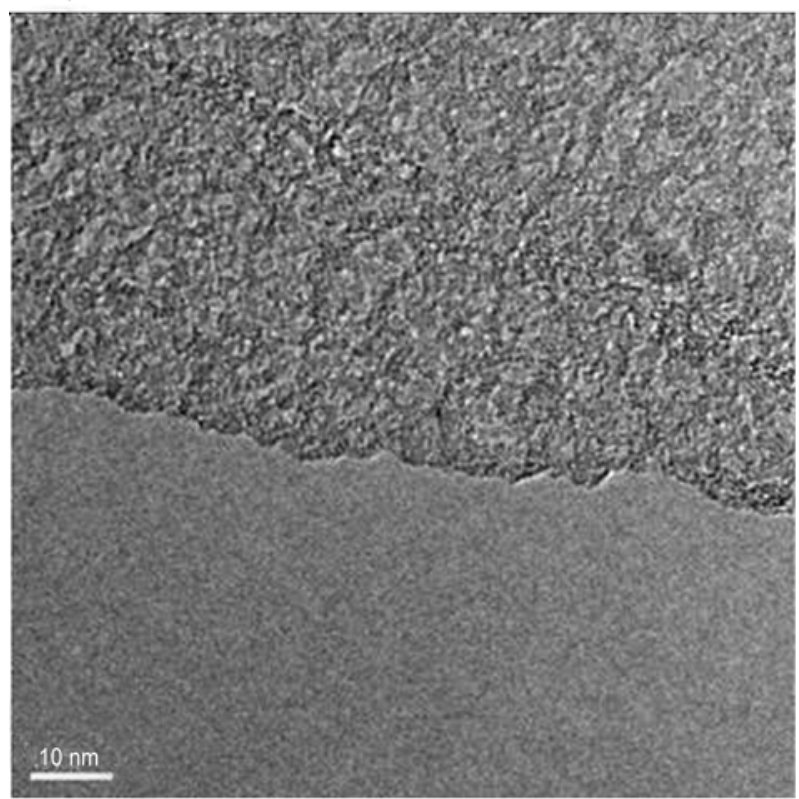

c) graphene is lower in the case of R-2 (ratio 0.82, $L a=3.6 \mathrm{~nm}$ ) which indicates less in-plane crystal size of graphene than in the case of R-1 (ratio 1.22, $L a=5.4 \mathrm{~nm}$ ). The $\mathrm{D}$ band intensity increases with the thinning of graphene and is absent for bulk graphite as defects can be observed more easily in thinner flakes [21]. So, more intense D band for nanocomposite film made by route 2 (R-2) can be attributed to better exfoliation of graphene in the polymer matrix. The reduction of graphene oxide before addition of polymer leads to re-agglomeration of graphene in route 1 while the polymer chains hinder the graphene to form stacking in the case of R-2. Hence, in situ reduction method (R-2) results

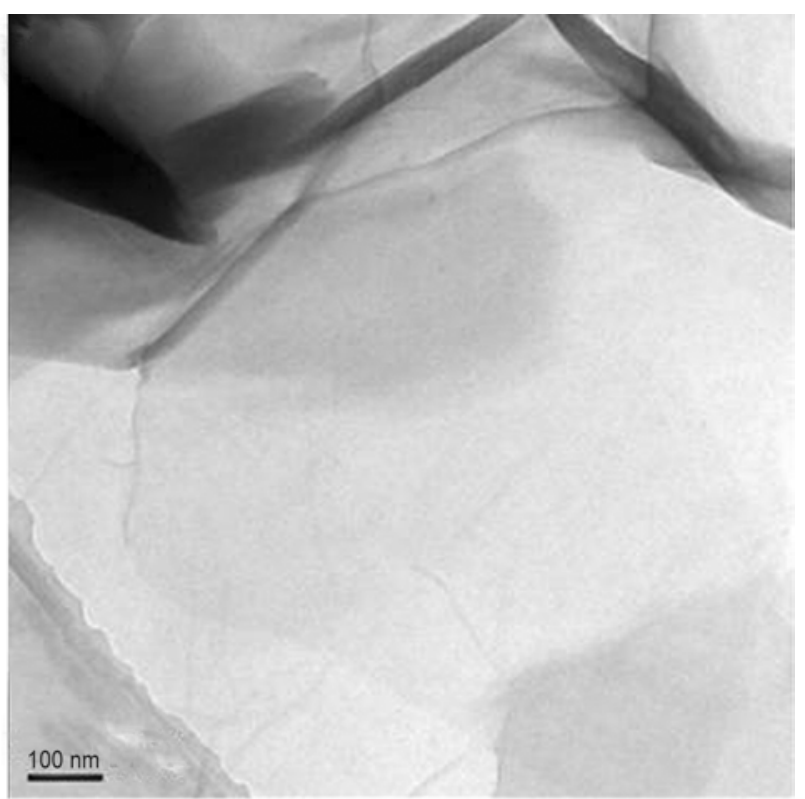

b)

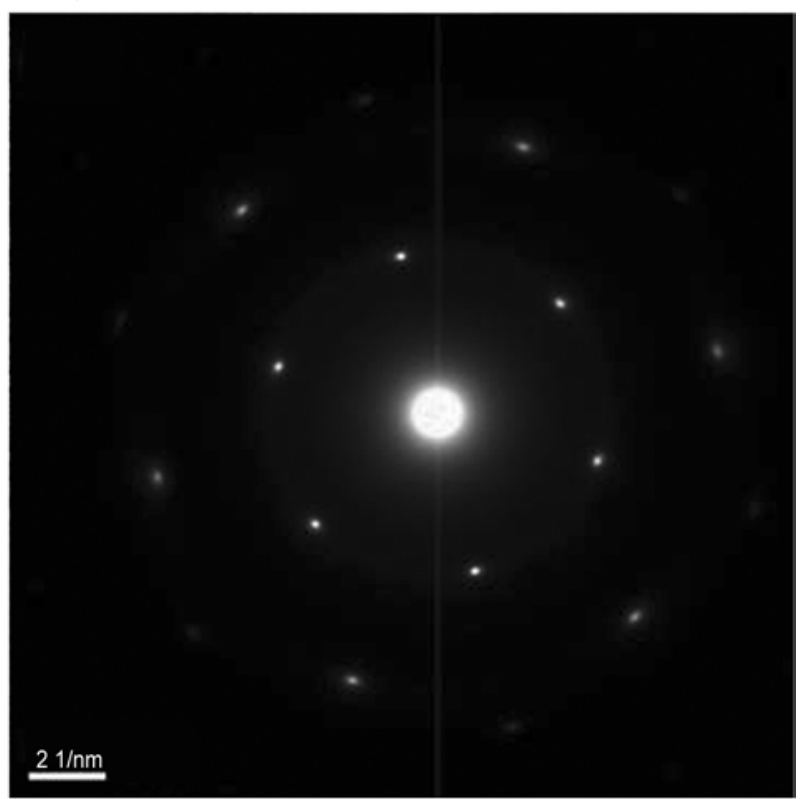

d)

Figure 3. TEM images of graphene oxide (a), graphene ((b) and (c)) and corresponding SAED pattern of graphene (d) 
in thinner graphene flakes and more dispersion of graphene in the polymer matrix which consequently shows more intense D band Raman peak as compared to pre-reduction method (R-1).

\subsubsection{Transmission electron microscope (TEM)}

Figure 3 shows the transmission electron microscope images of graphene oxide and graphene. After reduction, graphene sheets are mostly monolayers. The SAED pattern (Figure 3d) taken on the area mentioned in Figure $3 \mathrm{c}$ confirms the monolayer graphene [22, 23]. The synthesized graphene is folded and crinkled at different places. Existence of

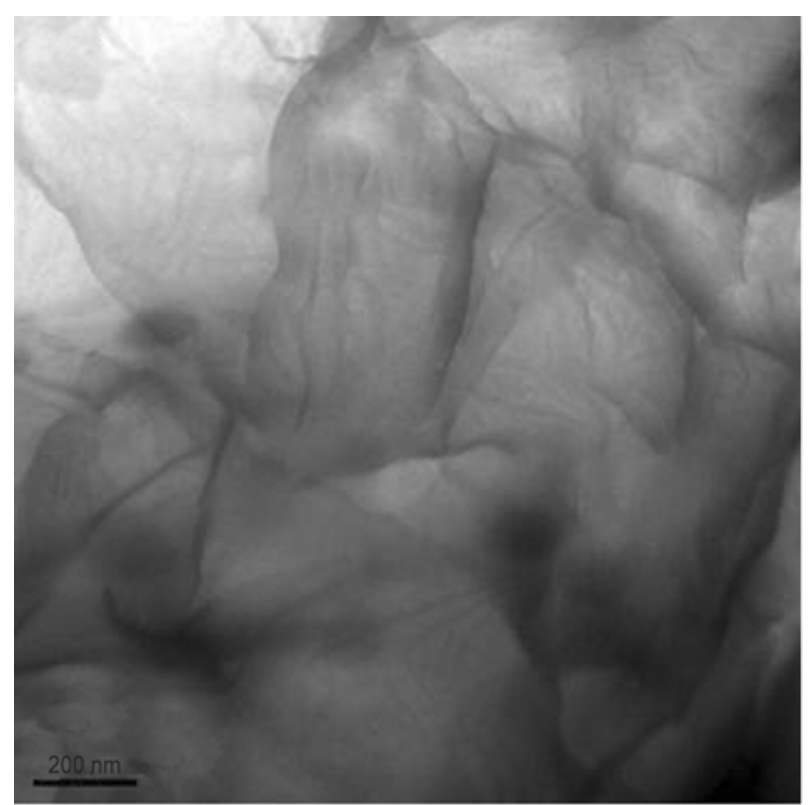

a)

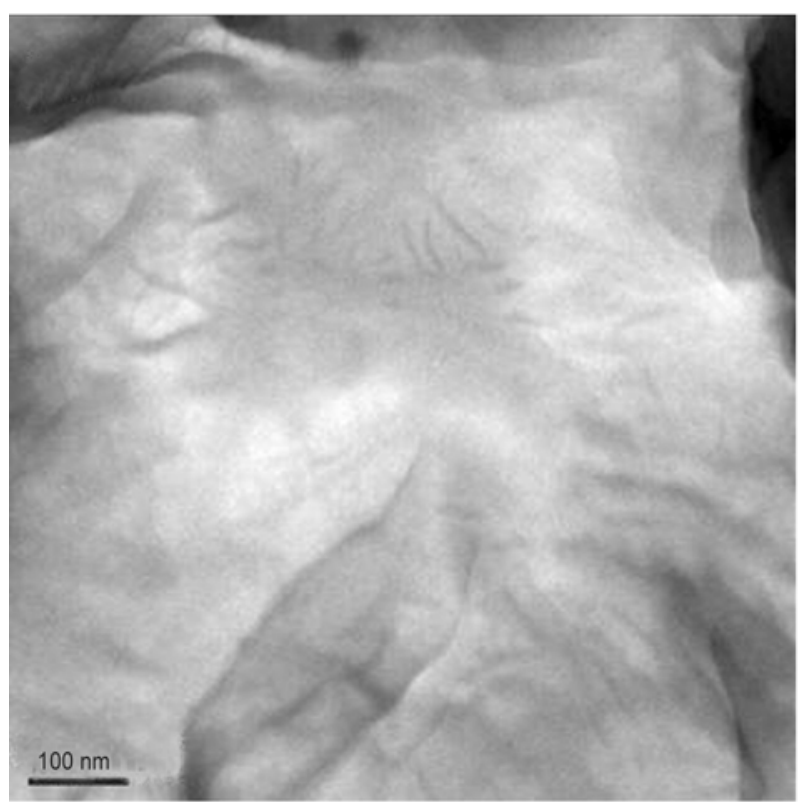

c) bi- or tri- layered flakes is also observed from SAED pattern taken in other areas. Hence, it can be concluded that the synthesized graphene is mostly monolayer while some few layered graphene also exist. The few layered graphene should also exfoliate to monolayer as it experiences further ultrasonic treatment and stirring during nanocomposite preparation.

TEM images and corresponding SAED patterns of the graphene/UHMWPE nanocomposites produced by two routes have been shown in Figure 4. The thin dark lines observed throughout the scanned area of both nanocomposite films (Figure 4a and 4c) can

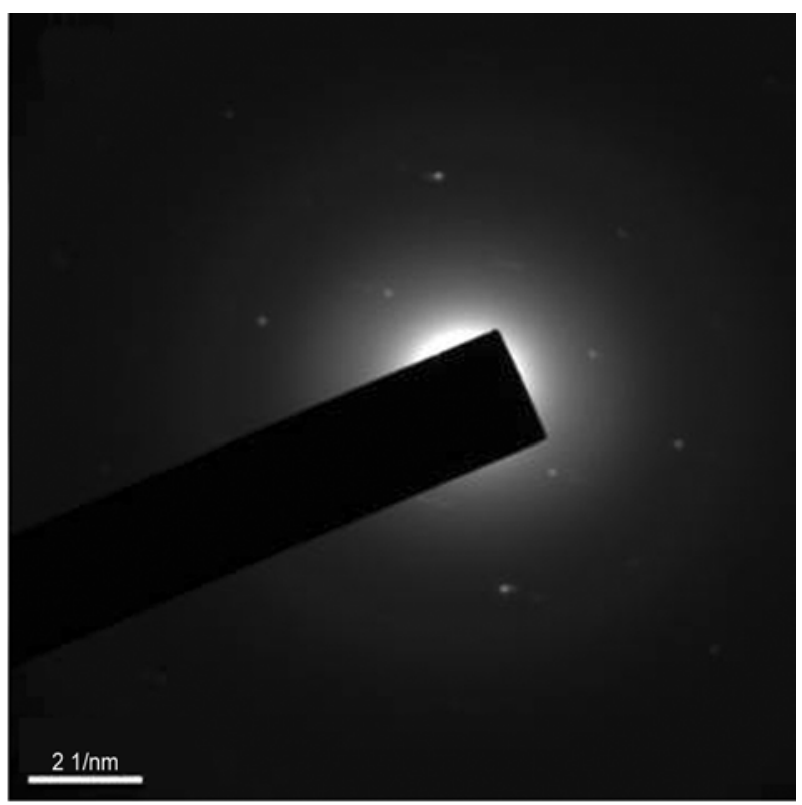

b)

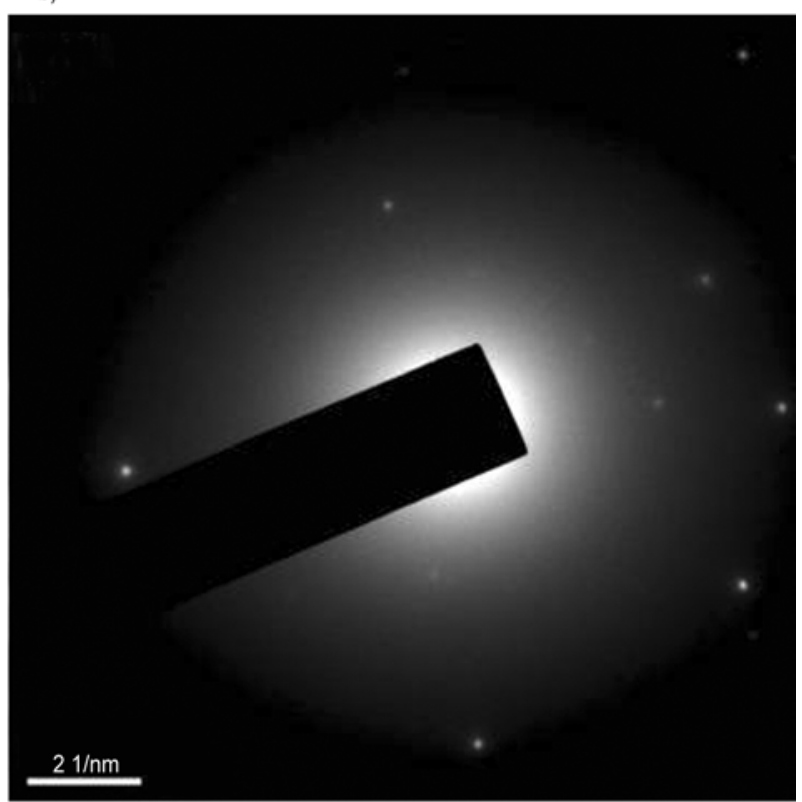

d)

Figure 4. TEM images ((a) and (c)) and corresponding SAED patterns of graphene-UHMWPE nanocomposites by R-1 ((a) and (b)) and R-2 ((c) and (d)) 
be attributed to graphene. However, it is noteworthy to mention that the crinkles developed by the ultra thin polymer film may also contribute to some of these lines. The TEM images show almost comparable dispersion of graphene in both the nanocomposites. From the Raman $\mathrm{G}$ band to $\mathrm{D}$ band ratio, R-2 or in situ reduction method results in thinner graphene flakes, hence, better dispersion of graphene in the resulted nanocomposite film. The SAED patterns reveal the difference in crystalline content. The patterns confirm the semi-crystalline nature of nanocomposite film produced by R-1 or pre-reduction method (Figure $4 \mathrm{~b}$ ) while amorphous content is very high in R-2 (Figure 4d). So, it can be concluded from the intensity of crystal spots and diffused amorphous rings that the crystalline content is less for in situ reduced nanocomposite film.

\subsubsection{Wide angle $X$-ray}

Figure 5 shows the wide angle X-ray curves of the three samples. The crystalline content estimated from the curves is $39.8 \%$ for pure UHMWPE film and almost same in $1 \mathrm{wt} \%$ graphene dispersed nanocomposite film produced through $\mathrm{R}-1$ (39.6\%). However, it is reduced to $32 \%$ in nanocomposite film produced by R-2. This may be attributed to the better exfoliated state of graphene which restricts the polymer chains to organize in particular arrange-

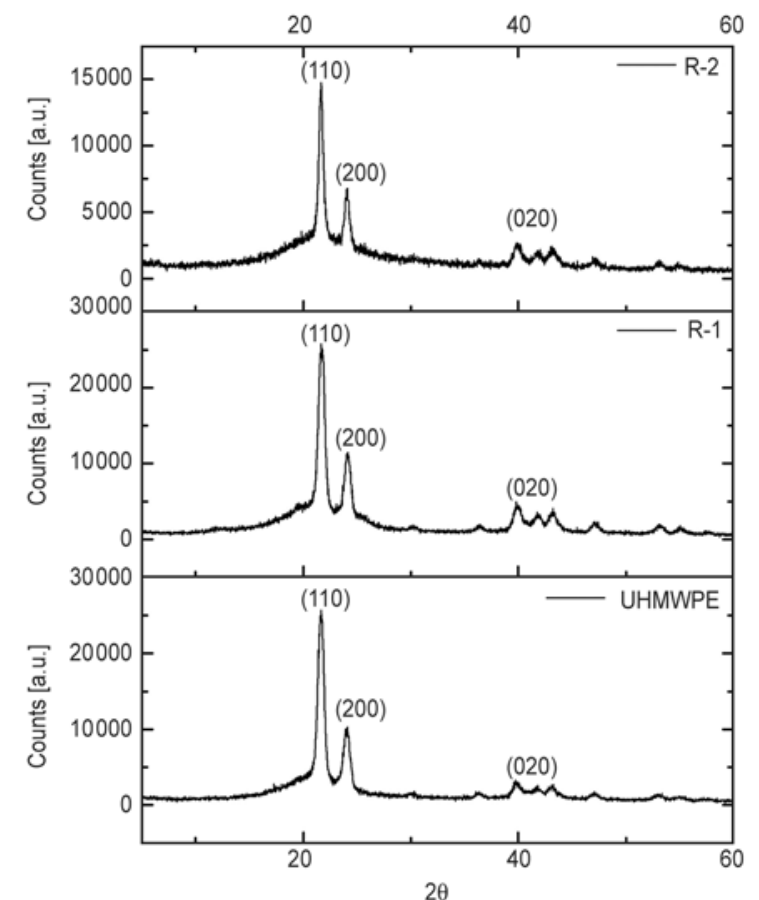

Figure 5. Wide angle X-ray curves for UHMWPE film, nanocomposite films produced by route $1(\mathrm{R}-1)$ and route $2(\mathrm{R}-2)$ ment and hinders the crystallization of polymer chains.

\subsubsection{Differential scanning calorimetry (DSC)}

Considering the heat of fusion for $100 \%$ crystalline UHMWPE as $289 \mathrm{~J} / \mathrm{g}$ [24], the crystallinity results can be obtained from the DSC curves. The estimated crystalline content for pure UHMWPE film is $41 \%$, for nanocomposite film produced by $\mathrm{R}-1$ is $39 \%$ and for nanocomposite film produced by R-2 is $35 \%$ as calculated from the DSC curves shown in Figure 6. The results are almost similar as that of X-ray crystallinity data. So, the graphene obstructs the crystal formation of polymer chains in nanocomposite film produced by in situ reduction method or R-2. Peak melting temperature is almost same in all three films, however, the onset of melting peak shifted to higher side in the case of R-1 and the peak width is much less, which indicates less variation in crystal size for pre-reduction method or R-1.

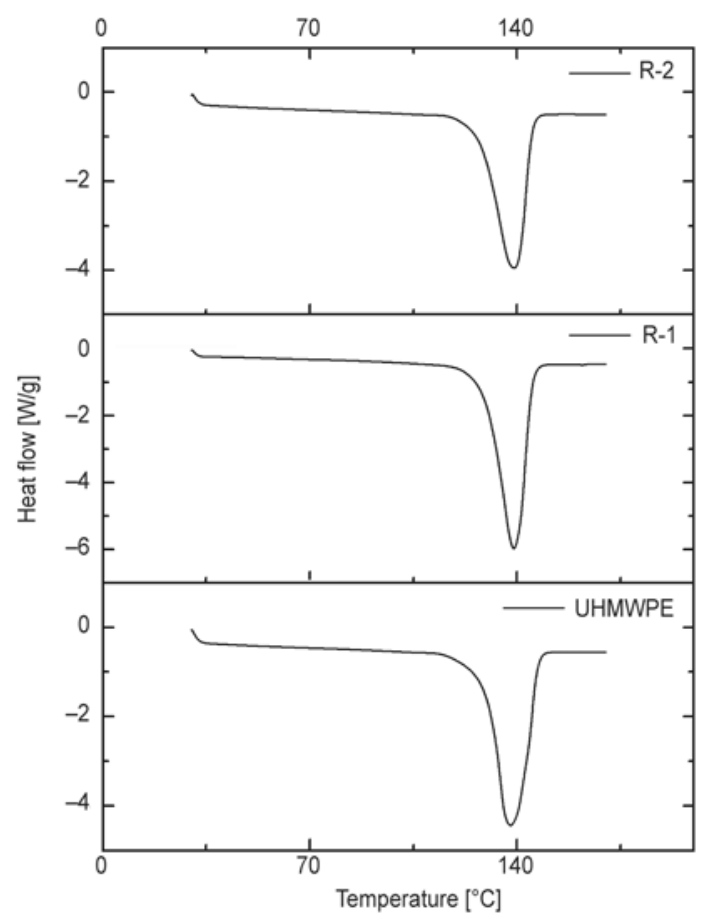

Figure 6. DSC curves for UHMWPE film, nanocomposite films produced by route $1(\mathrm{R}-1)$ and route $2(\mathrm{R}-2)$

\subsection{Mechanical behavior of graphene- UHMWPE nanocomposites}

\subsubsection{Tensile test}

The tensile test results are shown in Table 1. The tensile modulus calculated at initial straining (from 0.5 to $0.7 \%$ ) shows good increase in $\mathrm{R}-1$ or pre reduced graphene based nanocomposite film than 
Table 1. Tensile test results of pure and nanocomposite films (average of 10 readings with standard error)

\begin{tabular}{|l|c|c|c|}
\hline \multicolumn{1}{|c|}{ Sample name } & $\begin{array}{c}\text { Modulus [MPa] } \pm \text { standard } \\
\text { error }(\mathbf{n}=\mathbf{1 0})\end{array}$ & $\begin{array}{c}\text { Maximum strength [MPa] } \pm \\
\text { standard error }(\mathbf{n}=\mathbf{1 0})\end{array}$ & $\begin{array}{c}\text { Maximum strain [\%] } \pm \text { standard } \\
\text { error }(\mathbf{n}=\mathbf{1 0})\end{array}$ \\
\hline UHMWPE & $864 \pm 7$ & $12.6 \pm 2.0$ & $58 \pm 5$ \\
\hline R-1 & $1236 \pm 17$ & $22.2 \pm 1.5$ & $28 \pm 4$ \\
\hline R-2 & $416 \pm 23$ & $17.1 \pm 2.0$ & $41 \pm 5$ \\
\hline
\end{tabular}

the pure one (from 864 to $1236 \mathrm{MPa}$ ). However, it is decreased significantly in R-2 produced nanocomposite film. In both the process routes, use of $1 \mathrm{wt} \% \mathrm{GO}$ significantly improves the tensile strength of the UHMWPE and reduces the breaking extension. R-1 supersedes R-2 in terms of strength; however, the strain to break is also reduced. As compared to the pure film, the maximum strength has been increased almost twice in nanocomposite film produced by pre-reduction method or R-1 (from 12.6 to $22.2 \mathrm{MPa}$ ) and the strain is reduced to 28 from $58 \%$. For nanocomposite film by R-2, the strain is reduced to $41 \%$ with some improvement in strength (17.1 MPa). So, network hardening of polymer matrix is introduced by graphene in both nanocomposite films and the effect is more prominent in the case of $\mathrm{R}-1$. The results match with the observations made during characterization of the film. The low tensile modulus, high extension and low strength improvement are attributed to the lower crystalline content of nanocomposite film prepared by in situ reduction (R-2) while the high crystallinity as well as the influence of graphene leads to very high reduction in maximum strain for R-1 nanocomposite film. Representative tensile test curves of three types of samples are shown in Figure 7. As the films are not drawn, plateau zones are observed after maximum stress till they fail. The zone length is significantly reduced in both nanocomposite films which indicate network hardening at high stress level; but, R-2 shows more extension before stress relaxation near break. The tensile modulus (measured at low stress level) of in situ reduced graphene based nanocomposite film is even less than the pure UHMWPE film because of its higher amorphous content. The chain stretching and reordering happen only at higher stress level where the strength contribution of graphene results in higher tensile strength of the nanocomposite film (produced by R-2) than the pure one. Hence, the in situ reduced nanocomposite film has more ductility and significantly less modulus than the pre-reduced one. The incorporation of graphene in polymer matrix by R-2 method inhibits the polymer crystallization, thus lowering the crys-

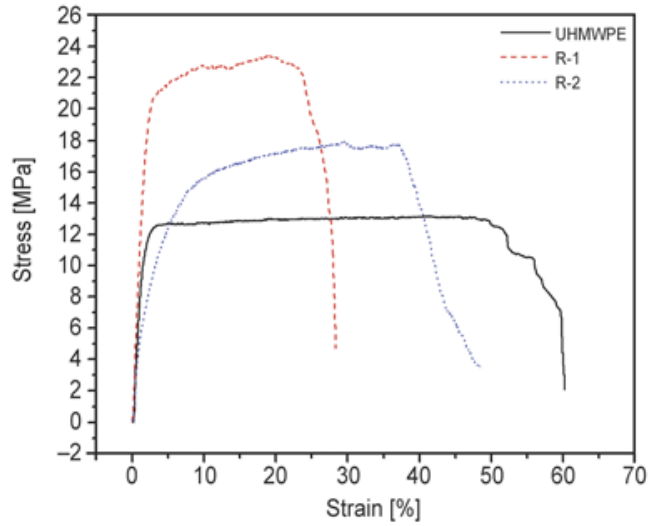

Figure 7. Representative stress-strain curve of UHMWPE film, nanocomposite films by R-1 and R-2

talline content which causes less strength improvement, less modulus and more ductility as compared to nanocomposite films produced by R-1 method.

\subsubsection{Creep test}

Creep tests have been carried out at different loading on films upto $72 \mathrm{~h}$ as shown in Figure 8. The test results are coded as ' $\mathrm{X}-\mathrm{Y}$ ' where $\mathrm{X}$ is sample name (coded as PE for UHMWPE film, R-1 for nanocomposite film produced by pre-reduction or route 1 method and R-2 for nanocomposite film produced by in situ or route 2 method) and $\mathrm{Y}$ is load percentage of the average maximum load (as of Table 1) applied on the sample throughout the creep test. The results show that at $20 \%$ loading, strains of pure and nanocomposite films are almost comparable (Figure 8a) at the beginning while over the extended period of time the strain is very high for pure film. The nanocomposite films produced by R-1 and R-2 shows less creep strain. The difference is more prominent at $40 \%$ loading (Figure $8 \mathrm{~b}$ ). The tendency to creep strain is very high for pure UHMWPE film as compared to nanocomposite films at that laoding. The increase in load to $60 \%$ of the breaking load leads to failure in $4 \mathrm{~h}$ in the case of pure film, while nanocomposite films fail after $24 \mathrm{~h}$ (Figure $8 \mathrm{c}$ ). The strain on creep is much less in the case of both nanocomposite films, however, nanocomposite film produced by pre-reduction or $\mathrm{R}-1$ method shows better results than in situ reduced (R-2) 

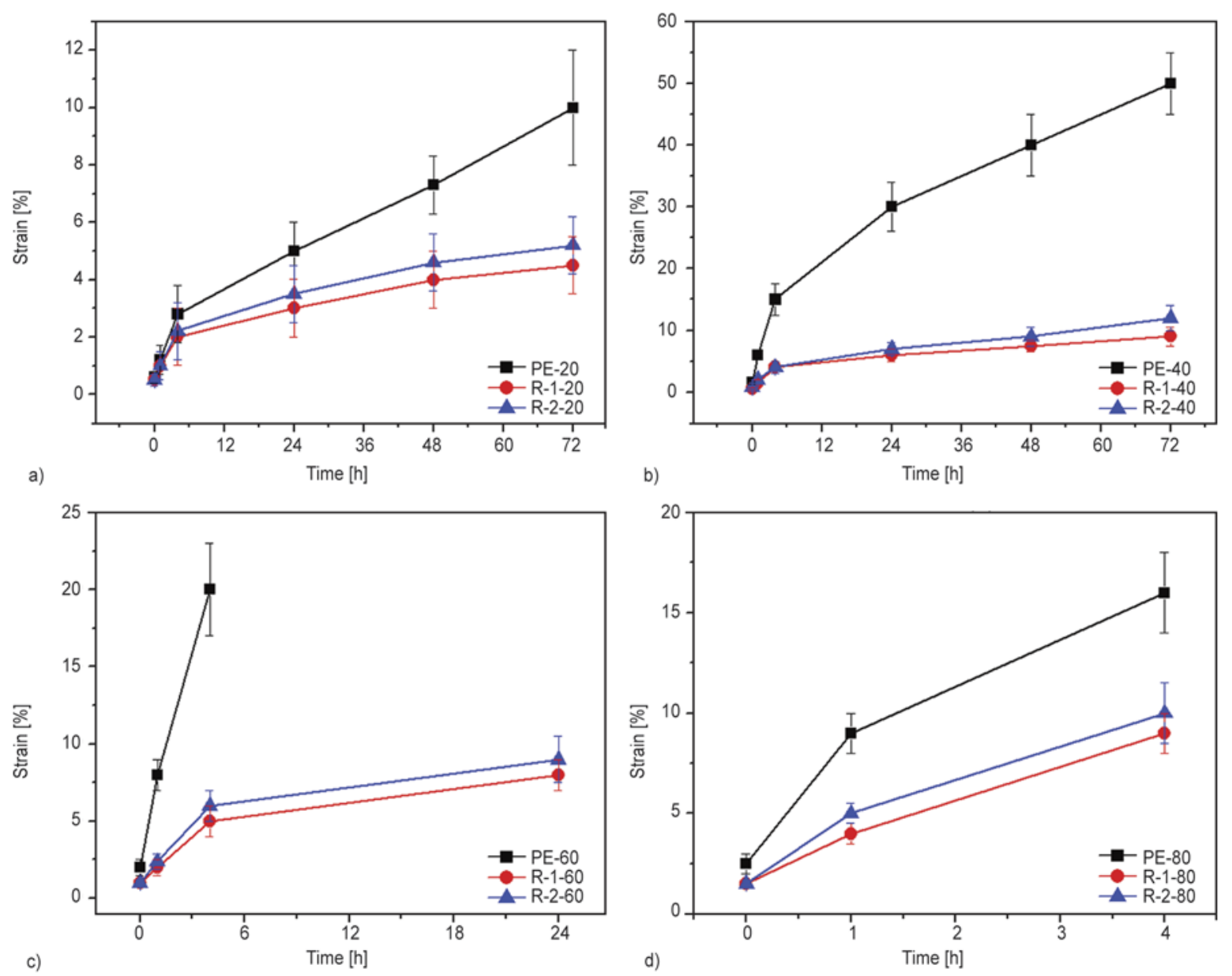

Figure 8. Strain of pure films (PE) and nanocomposite films from route 1 (R-1) and route 2 (R-2) with time under $20 \%$ (a), $40 \%$ (b), $60 \%$ (c) and $80 \%$ (d) load of average maximum load

one at all loadings. The results are similar in nature to tensile test results and hence, it can be concluded that the polymer network hardening is the reason behind the low creep for the nanocomposite films. The strain rate is low for the samples undergoing the such creep test. Hence, polymer samples get sufficient time to reorder its chains, distribute the stress and transfer the load to the reinforcing nanoparticles. This leads to significant improvement in reduction of creep strain for both the nanocomposite films.

A scheme to explain the morphology and properties of nanocomposite films is shown in Figure 9. All the solution cast films are semi crystalline in nature, however, the crystals are not oriented to a particular direction as there is no stretching or drawing. The crystals grow with the evaporation of solvents till they are obstruced either by neighbouring crystals or by nanoparticles (here graphene nanoplatelets). The process continues till complete solidification. The entangled molecular chains having no particu-
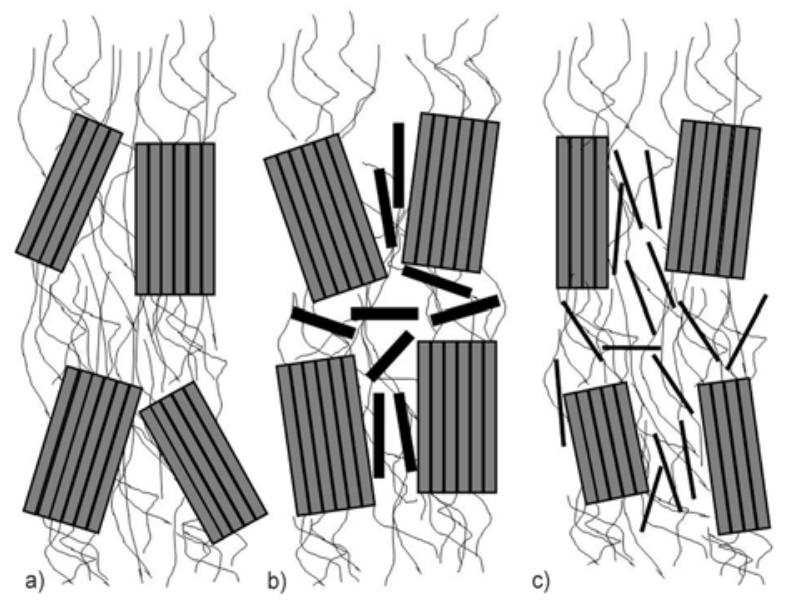

Figure 9. Schematic of proposed morphology for pure UHMWPE film (a), nanocomposite film by prereduction method (b) and nanocomposite film by in situ reduction method (c)

lar orientation contribute to its amorphous fraction. The nanoparticles reinforce the amorphous region of polymer nanocomposites. In the case of prereduced graphene dispersed nanocomposite film, the graphene nanoplatelets are well distributed in 
the polymer solution, hence, the size distribution of crystals are less as compared to pure one as shown in Figure $9 \mathrm{~b}$. The better or fully exfoliated graphene has higher surface area than the less exfoliated one, so it restricts the polymer chains more than the multilayer graphene sheets. The interference of graphene prevents the polymer chains to form the regular crystalline array as shown in Figure 9c. When subjected to tensile testing, the entangled polymer chains in amorphous region get stretched and the load is transferred to nanoparticles which leads to increase in maximum strength. However, the ductility of the film is reduced significantly as the chain mobility is restricted. The high amorphous content of the in situ reduced nanocomposite film leads to less tensile modulus and more ductility as compared to pre-reduced one. The ductility of R-2 produced nanocomposite film never achieves the level of pure UHMWPE film due to the nanofiller constrain and the tensile molulus at low stress level is also less as it is less cryslline in nature.

\section{Conclusions}

Graphene can be used as reinforcement for UHMWPE to improve its tensile property and to reduce its creep behavior. The dispersion of graphene in polymer matrix by the pre-reduction method may not be as good as in situ reduction method, but the latter leads to significant loss in crystallinity due to restriction in polymer chains movements by the exfoliated graphene flakes. In the in situ reduction process, the exfoliated graphene impedes the crystalline arrangement for the polymer which in turn decreases the tensile modulus, increases strain at break as well as the creep strain as compared to prereduced graphene based nanocomposite. The network hardening of matrix is more in pre-reduction or the first route of nanocomposite film production (strain reduced to 28\%). The strength enhancement in first route (more than $76 \%$ improvement as compared to pure film) is also much better than the second route due to reinforcement by graphene as well as higher crystalline content. Hence, the crystalline content is also a significant contributing factor for improvement in tensile properties along with the dispersion of graphene. It can be concluded that the pre-reduction method is suitable for high strength and creep resistant applications while in situ reduc- tion method may perform better for applications where more ductility is required. The underlying mechanism of graphene dispersion at its different concentration needs to be explored by further characterizations. More investigation in this route by using functionalized graphene and drawing of films will be expected and interesting.

\section{Acknowledgements}

Authors like to express their heartiest acknowledgement to Dr. Baldev Raj, President Research, PSG Institutions, for his continuous encouragement and help to facilitate this work. Authors also express their deep gratitude to management of PSG Institutions and College of Materials and Engineering (CMSE), Donghua University for their support as well as the financial support from the National Natural Science Foundation for Distinguished Young Scholar of China (50925312) and Natural Science Foundation of China (51273040).

\section{References}

[1] Kavesh S., Prevorsek D. C.: Ultra high strength, high modulus polyethylene spectra fibers and composites. International Journal of Polymeric Materials and Polymeric Biomaterials, 30, 15-56 (1995).

DOI: $10.1080 / 00914039508031459$

[2] Li C. S., Zhan M. S., Huang X. C., Zhou H.: Degradation behavior of ultra-high molecular weight polyethylene fibers under artificial accelerated weathering. Polymer Testing, 31, 938-943 (2012).

DOI: 10.1016/j.polymertesting.2012.06.009

[3] Bracco P., Brunella V., Luda M. P., Zanetti M., Costa L.: Radiation-induced crosslinking of UHMWPE in the presence of $c o$-agents: Chemical and mechanical characterisation. Polymer, 46, 10648-10657 (2005). DOI: $10.1016 /$ j.polymer.2005.08.095

[4] Ramanathan T., Abdala A. A., Stankovich S., Dikin D. A., Herrera-Alonso M., Piner R. D., Adamson D. H., Schniepp H. C., Chen X., Ruoff R. S., Nguyen S. T., Aksay I. A., Prud'Homme R. K., Brinson L. C.: Functionalized graphene sheets for polymer nanocomposites. Nature Nanotechnology, 3, 327-331 (2008). DOI: $10.1038 /$ nnano.2008.96

[5] Coleman J. N., Khan U., Blau W. J., Gun'ko Y. K.: Small but strong: A review of the mechanical properties of carbon nanotube-polymer composites. Carbon, 44, 1624-1652 (2006). DOI: 10.1016/j.carbon.2006.02.038

[6] Andrews R., Weisenberger M. C.: Carbon nanotube polymer composites. Current Opinion in Solid State and Materials Science, 8, 31-37 (2004). DOI: $10.1016 / \mathrm{j} . \operatorname{cossms} .2003 .10 .006$ 
[7] Ma P-C., Siddiqui N. A., Marom G., Kim J-K.: Dispersion and functionalization of carbon nanotubes for polymer-based nanocomposites: A review. Composites Part A: Applied Science and Manufacturing, 41, 13451367 (2010).

DOI: 10.1016/j.compositesa.2010.07.003

[8] Kuilla T., Bhadra S., Yao D., Kim N. H., Bose S., Lee J. H.: Recent advances in graphene based polymer composites. Progress in Polymer Science, 35, 1350 1375 (2010). DOI: 10.1016/j.progpolymsci.2010.07.005

[9] Arai T., Tominaga Y., Asai S., Sumita M.: A study on correlation between physical properties and interfacial characteristics in highly loaded graphite-polymer composites. Journal of Polymer Science Part B: Polymer Physics, 43, 2568-2577 (2005).

DOI: $10.1002 /$ polb.20543

[10] Song P., Cao Z., Cai Y., Zhao L., Fang Z., Fu S.: Fabrication of exfoliated graphene-based polypropylene nanocomposites with enhanced mechanical and thermal properties. Polymer, 52, 4001-4010 (2011).

DOI: $10.1016 /$ j.polymer.2011.06.045

[11] Yoon O. J., Jung C. Y., Sohn I. Y., Kim H. J., Hong B., Jhon M. S., Lee N-E.: Nanocomposite nanofibers of poly(D, L-lactic-co-glycolic acid) and graphene oxide nanosheets. Composites Part A: Applied Science and Manufacturing, 42, 1978-1984 (2011).

DOI: $10.1016 /$ j.compositesa.2011.08.023

[12] Sengupta R., Bhattacharya M., Bandyopadhyay S., Bhowmick A. K.: A review on the mechanical and electrical properties of graphite and modified graphite reinforced polymer composites. Progress in Polymer Science, 36, 638-670 (2011).

DOI: $10.1016 /$ j.progpolymsci.2010.11.003

[13] Jiang X., Drzal L. T.: Improving electrical conductivity and mechanical properties of high density polyethylene through incorporation of paraffin wax coated exfoliated graphene nanoplatelets and multi-wall carbon nano-tubes. Composites Part A: Applied Science and Manufacturing, 42, 1840-1849 (2011).

DOI: $10.1016 /$ j.compositesa.2011.08.011

[14] Hummers Jr. W. S., Offeman R. E.: Preparation of graphitic oxide. Journal of the American Chemical Society, 80, 1339 (1958).

DOI: $10.1021 / \mathrm{ja} 01539 \mathrm{a} 017$

[15] Dreyer D. R., Park S., Bielawski C. W., Ruoff R. S.: The chemistry of graphene oxide. Chemical Society Reviews, 39, 228-240 (2010).

DOI: $10.1039 / \mathrm{B} 917103 \mathrm{G}$
[16] Bahr J. L., Mickelson E. T., Bronikowski M. J., Smalley R. E., Tour J. M.: Dissolution of small diameter single-wall carbon nanotubes in organic solvents? Chemical Communications, 2, 193-194 (2001). DOI: 10.1039/B008042J

[17] Hamilton C. E., Lomeda J. R., Sun Z., Tour J. M., Barron A. R.: High-yield organic dispersions of unfunctionalized graphene. Nano Letters, 9, 3460-3462 (2009). DOI: $10.1021 / \mathrm{n} 19016623$

[18] Narh K. A., Barham P. J., Keller A.: Effect of stirring on the gelation behavior of high-density polyethylene solutions. Macromolecules, 15, 464-469 (1982). DOI: $10.1021 / \mathrm{ma} 00230 \mathrm{a} 051$

[19] Subrahmanyam K. S., Vivekchand S. R. C., Govindaraj A., Rao C. N. R.: A study of graphenes prepared by different methods: Characterization, properties and solubilization. Journal of Materials Chemistry, 18, 15171523 (2008).

DOI: 10.1039/B716536F

[20] Bakshi S. R., Tercero J. E., Agarwal A.: Synthesis and characterization of multiwalled carbon nanotube reinforced ultra high molecular weight polyethylene composite by electrostatic spraying technique. Composites Part A: Applied Science and Manufacturing, 38, 24932499 (2007).

DOI: 10.1016/j.compositesa.2007.08.004

[21] Ni Z., Wang Y., Yu T., Shen Z.: Raman spectroscopy and imaging of graphene. Nano Research, 1, 273-291 (2008).

DOI: $10.1007 / \mathrm{s} 12274-008-8036-1$

[22] Suk J. W., Kitt A., Magnuson C. W., Hao Y., Ahmed S., An J., Swan A. K., Goldberg B. B., Ruoff R. S.: Transfer of CVD-grown monolayer graphene onto arbitrary substrates. ACS nano, 5, 6916-6924 (2011).

DOI: $10.1021 / \mathrm{nn} 201207 \mathrm{c}$

[23] Ferrari A. C., Meyer J. C., Scardaci V., Casiraghi C., Lazzeri M., Mauri F., Piscanec S., Jiang D., Novoselov K. S., Roth S., Geim A. K.: Raman spectrum of graphene and graphene layers. Physical Review Letters, 97, 187401/1-187401/4 (2006).

DOI: 10.1103/PhysRevLett.97.187401

[24] Andjelić S., Richard R. E.: Crystallization behavior of ultrahigh molecular weight polyethylene as a function of in vacuo $\gamma$-irradiation. Macromolecules, 34, 896906 (2001).

DOI: $10.1021 / \mathrm{ma} 0008241$ 